\title{
SALT Spectropolarimetry and Self-Consistent SED and Polarization Modeling of Blazars*
}

\section{Markus Böttcher ${ }^{\dagger}$, Hester Schutte}

Centre for Space Research, North-West University, Potchefstroom, South Africa

E-mail: Markus.Bottcher@nwu.ac.za

\section{Brian van Soelen, Richard J. Britto $\ddagger$ Johannes P. Marais}

Department of Phyics, Bloemfontein, South Africa

\section{David A. H. Buckley}

South African Astronomical Observatory, Cape Town, South Africa

\begin{abstract}
We report on results from a target-of-opportunity program to obtain optical spectropolarimetry of flaring $\gamma$-ray blazars with the Southern African Large Telescope (SALT). SALT spectropolarimetry and contemporaneous multi-wavelength spectral energy distributions (SEDs) are modelled self-consistently with a leptonic single-zone model. Such modeling provides an accurate estimate of the degree of order of the magnetic field in the emission region and the thermal contributions from the host galaxy and the accretion disk to the SED, thus putting strong constraints on the physical parameters of the gamma-ray emitting region. For the specific case of the $\gamma$-ray blazar $4 \mathrm{C}+01.02(z=2.1)$, which underwent a large $\gamma$-ray flare in July 2016, we demonstrate that the combined SED and spectropolarimetry modeling provides an estimate of the mass of the central black hole in this blazar to $M_{\mathrm{BH}} \sim 2 \times 10^{9} M_{\odot}$.
\end{abstract}

7th Fermi Symposium 2017

15-20 October 2017

Garmisch-Partenkirchen, Germany

*based on observations made with the Southern African Large Telescope (SALT) under programme 2016-2-LSP001 (PI: D. A. H. Buckley)

${ }^{\dagger}$ Speaker.

${ }^{\ddagger}$ for the Fermi-LAT collaboration 


\section{Introduction}

Blazars are the most abundant extragalactic source class detected by Fermi-LAT. Their spectral energy distribution (SED) is dominated by two broad non-thermal components. The low-frequency component is synchrotron radiation from relativistic electrons, while for the high-energy component both leptonic and hadronic emission mechanisms are possible (e.g., [4, 5]). In leptonic models, the high-energy emission is produced by Compton scattering of soft radiation fields off relativistic electrons $[4,10,5]$. In the infrared through UV, also thermal components from the host galaxy and the accretion-disk, broad-line-region (BLR) and dust-torus system add to the SED. There is significant ambiguity concerning the underlying particle distributions and the location of and physical conditions within the $\gamma$-ray emission zone (e.g., $[10,5]$ ), which can generally not be broken by SED modeling alone.

In this paper, we demonstrate how optical polarization can help to break some of these degeneracies (see also, $[11,19,20])$. The optical emission from blazars is often dominated by polarized jet synchrotron emission. Additional contributions to the optical spectrum may arise from the host galaxy, a dust torus, and from the accretion disk. These thermal contributions are expected to be unpolarized and will reveal their presence through a decline of the degree of polarization (compared to pure synchrotron emission) in spectropolarimetric observations (e.g., [17, 12]).

The above considerations motivated us to use the Southern African Large Telescope (SALT) for spectropolarimetry of flaring $\gamma$-ray blazars to constrain the contribution of thermal components to the optical spectrum. We focus on the blazar 4C+01.02, a high-polarization flat-spectrum radio quasar (FSRQ) at a redshift of $z=2.1$. which underwent a large $\gamma$-ray flare in July 2016 [6]. The resulting SEDs and spectropolarimetry results were modelled with a leptonic single-zone model that self-consistently calculates the SED along with the expected optical spectropolarimetry signatures (see Section 3).

\section{Observations}

Our observations were conducted in the framework of a SALT Large Program on high-energy transients (PI: D. Buckley). This program aims at target-of-opportunity (ToO) spectroscopy and spectropolarimetry observations of various transients, including flaring blazars. Within the first year of this program (April 2016 - May 2017), 14 ToO spectropolarimetry observations targeting 7 different blazars were conducted, revealing generally frequency-dependent polarization degrees in the range $\sim 5-25 \%$. In the following we focus on the particularly interesting case of the FSRQ $4 \mathrm{C}+01.02(\mathrm{PKS}$ B0106+013; $z=2.1$ ).

\subsection{Fermi-LAT}

We analysed Fermi-LAT data from 4C+01.02 between $100 \mathrm{MeV}$ and $300 \mathrm{GeV}$ during its high state in 2016, using the Pass 8 data representation [2] and the Fermi Science Tools version v10r0p5. ${ }^{1}$ We applied the following standard analysis cuts: radius of the Region of interest $(\mathrm{ROI})=15^{\circ}$; Source region $=\mathrm{ROI}+10^{\circ}$; sOURCE class; front + back event type; zenith angle $<90^{\circ}$;

\footnotetext{
${ }^{1}$ http://fermi.gsfc.nasa.gov/ssc/data/analysis/
} 


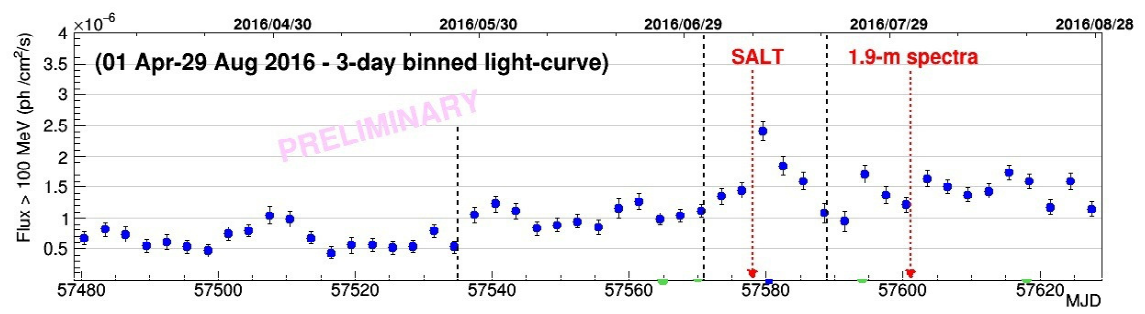

Figure 1: 3-day binned Fermi-LAT light curve at $E>100 \mathrm{MeV}$ of $4 \mathrm{C}+01.02$ for 2016 April - August. The vertical dashed lines demarcate the period July $2-20$, used for the extraction of the spectral points in Fig. 3.

DATA_QUAL=1, LAT_CONFIG=1; Diffuse emission: gll_iem_v06.fits (Galactic) and iso_P8R2_SOURCE_V6_v06.txt (extragalactic) templates. In the light-curve processing, we used the unbinned likelihood gtlike/pyLikelihood tool, and modeled the source of interest with a single power law spectrum. In the SED processing, we used the binned likelihood analysis within the Enrico Python package [14]. Figure 1 shows the light curve of 4C+01.02 during 1 April - 29 August, 2016. The July $2-20$ period contains the highest peak flux for the considered high state, which also was the highest flux ever detected with Fermi-LAT for this source to date (daily average $F_{>100 \mathrm{MeV}} \simeq(2.8 \pm 0.3) \times 10^{-6} \mathrm{ph} \mathrm{cm}^{-2} \mathrm{~s}^{-1}$ on 10 July [MJD 57579]). The SED of the main flare is plotted in Fig. 3 along with multiwavelength data.

\subsection{SALT}

SALT is a 10-m class telescope, located at the South African Astronomical Observatory (SAAO), near Sutherland, South Africa. One of its main instruments, the Robert Stobie Spectrograph (RSS), is capable of performing spectroscopy in various modes and is sensitive to wavelengths from $\sim 3200 \AA$ to $\geq 9000 \AA$ [8]. The results presented in Fig. 2 were obtained using RSS in the spectropolarimetry "LINEAR" mode [13] on 2016 July 9 and November 27, 28 and 29. Each spectrum, along with the polarization degree and polarization angle as a function of wavelength, was obtained through four exposures of $600 \mathrm{~s}$, one for each of the four orientations of the half-wave plates $\left(0^{\circ}, 45^{\circ}, 22.5^{\circ}\right.$ and $67.5^{\circ}$ respectively). Data reduction was performed using the POLSALT reduction pipeline, version $0.2 . \operatorname{dev} 0-$ py $2.7 .^{2}$

\subsection{LCO}

The Las Cumbres Observatory (LCO) is a global network of 18 telescopes located at 8 different locations on different continents in both hemispheres [7]. Observations were undertaken with the 1-m class telescopes using the SBIG CCD. Data reduction followed the standard procedures using the IRAF/NOAO packages. Differential photometry was performed using four nearby comparison stars whose magnitudes were taken from the NOMAD catalog [18]. The photometric data points included in Fig. 3 were taken on 2016, August 2.

\footnotetext{
${ }^{2}$ https://github.com/saltastro/polsalt
} 
4C +01.02 - SALT-RSS - polarization binning: 300A

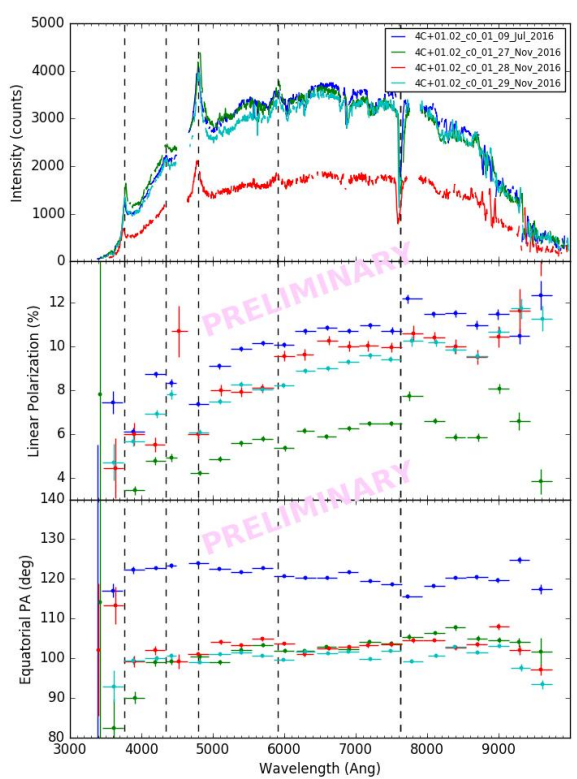

Figure 2. Top panel: SALT RSS count spectra of 4C+01.02 during four observing windows, including the 2016 July 9 flare indicated in Fig. 1 (blue). Dashed vertical lines indicate the positions of the following lines: Ly $\alpha 1216 \AA$, Si IV $1400 \AA$, C IV $1549 \AA$, C III] $1909 \AA$ and a telluric absorption line. Middle panel: Linear polarization degree as function of wavelength from SALT spectropolarimetry for the four SALT observing windows, as in the top panel. Bottom panel: Polarization angle as a function of wavelength for the four SALT observations.

\section{SED and Spectropolarimetry Modeling}

The simultaneous fitting of the SED and spectropolarimetry data on $4 \mathrm{C}+01.02$ is done by a combination of the leptonic single-zone model of [5] with a newly developed spectropolarimetry fitting routine. The code of [5] evaluates the non-thermal synchrotron and Compton radiation spectrum based on an equilibrium solution for the electron spectrum, assuming a rapid acceleration mechanism that provides a power-law injection spectrum with index $q$ between cut-off energies $\gamma_{\min }$ and $\gamma_{\max }$ that is balanced by self-consistent radiative losses and escape. This code is used to determine down a first iteration of the radiating electron distribution. A newly developed code (Schutte \& Böttcher 2018, in preparation) is then used for a combined fit of the radio - optical SED and the optical spectropolarimetry data. It calculates the synchrotron spectrum, based on the broken power-law distribution found from the SED fit and adds unpolarized contributions from a Shakura-Sunyaev [16] type accretion disk, $F_{\mathrm{AD}}$, and the prominent C IV and Ly $\alpha$ emision lines, $F_{\text {lines }}$ to the spectrum. The degree of polarization of the synchrotron emission is evaluated using the full energy integrals over the modified Bessel functions (Equ. 6.37 in [15]), with a correction factor $f_{\mathrm{B}, \text { order }}$ to account for not perfectly ordered $\mathrm{B}$-fields. The frequency-dependent degree of polarization of the total observed optical spectrum is then calculated as

$$
\Pi(v)=\frac{\Pi_{\mathrm{sy}}(v) F_{\mathrm{sy}}(v)}{F_{\mathrm{sy}}(v)+F_{\mathrm{AD}}(v)+F_{\text {lines }}(v)} .
$$

A simultaneous fit to the radio - optical SED and the optical spectropolarimetry data of 4C+01.02 during the 2016 July flare is obtained by adjusting the parameters of the radiating electron distribution, the B-field ordering parameter $f_{\mathrm{B}, \text { order }}$, and the black-hole mass and accretion-disk luminosity. Since only the optical and $\gamma$-ray data are contemporaneous, we include the archival Swift-XRT Xray spectrum from summed observations in 2007 and 2008 [9] as a guide for our spectral fit since they also correspond to a moderately high $\gamma$-ray flux state of the source. 

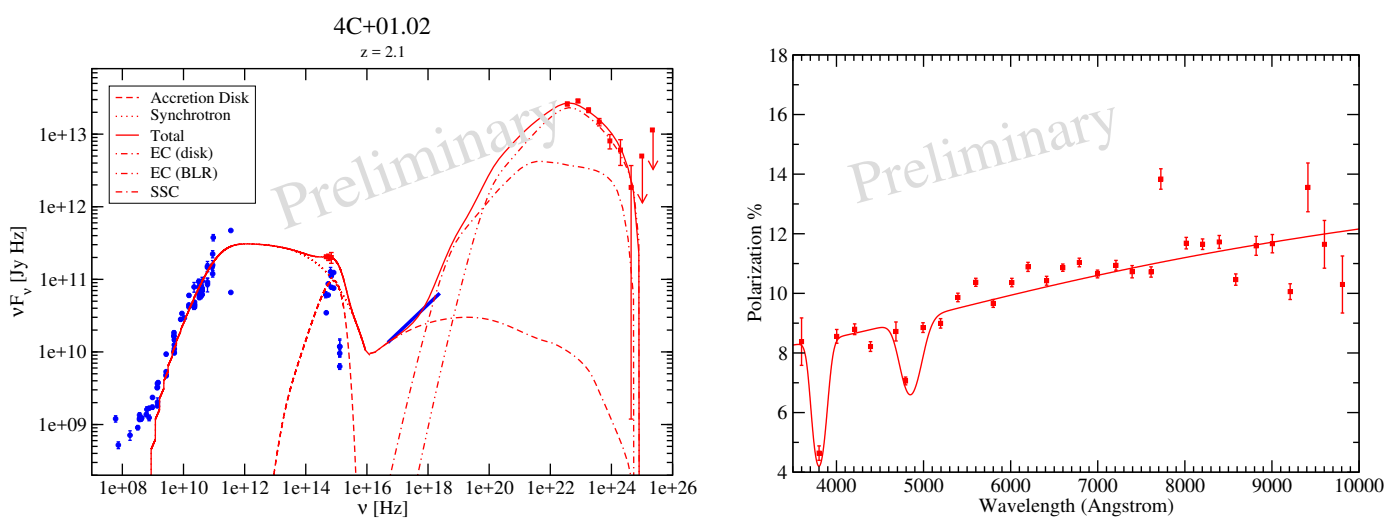

Figure 3: Left: Contemporaneous optical $+\gamma$-ray SED (red) of 4C+01.02, along with archival data (from $\mathrm{NED}^{3}$ - blue), and our SED model (solid red). Right: Fit to the SALT spectropolarimetry data.

\section{Results}

Figure 3 shows the result of our combined SED + spectropolarimetry fit to $4 \mathrm{C}+01.02$ in July 2016. The most relevant parameters used for this fit are listed in Table 1. All of the listed parameters are free parameters, varied to obtain a simultaneous fit to the SED and spectropolarimetry data, with the energy partition ratio being calculated from the kinetic and magnetic powers in the jet, which, in turn, are free input parameters.

Table 1: Model parameters.

\begin{tabular}{cc}
\hline Parameter & Value \\
\hline Kinetic power in radiating electrons $L_{e}$ & $2.5 \times 10^{45} \mathrm{erg} / \mathrm{s}$ \\
Magnetic field $B$ & $1 \mathrm{G}$ \\
Magnetic-field order $f_{\mathrm{B}, \text { order }}$ & 0.21 \\
Energy partition ratio $L_{B} / L_{e}$ & 0.45 \\
Emission-region distance from BH & $0.2 \mathrm{pc}$ \\
Black-hole mass $M_{\mathrm{BH}}$ & $2.4 \times 10^{9} M_{\odot}$ \\
Accretion-disk luminosity $L_{d}$ & $1.3 \times 10^{46} \mathrm{erg} / \mathrm{s}$ \\
External radiation field $u_{\mathrm{ext}}$ & $5 \times 10^{-3} \mathrm{erg} / \mathrm{cm}^{3}$ \\
\hline
\end{tabular}

The peak of the accretion-disk emission is well constrained by the spectropolarimetry results to be located near or beyond the blue end of the optical spectrum, requiring a central black-hole mass of $M_{\mathrm{BH}} \sim 2 \times 10^{9} M_{\odot}$.

\section{Summary and Conclusions}

We have presented results of $\mathrm{ToO}$ spectropolarimetry observations of the high-redshift FSRQ $4 \mathrm{C}+01.02$ during a large $\gamma$-ray flare in July 2016. The contemporaneous SED and spectropolarimetry data were fitted in a consistent way with a single-zone leptonic model. This combined fitting

\footnotetext{
${ }^{3}$ https://ned.ipac.caltech.edu/
} 
constrained the accretion-disk contribution to the SED and, in particular, the black-hole mass in the center of $4 \mathrm{C}+01.02$ to $M_{\mathrm{BH}} \sim 2 \times 10^{9} M_{\odot}$. This is in tension with the result of [9] who modelled the entire optical - UV spectrum of the source as dominated by accretion-disk emission, requiring a black-hole mass of $M_{\mathrm{BH}}=5 \times 10^{9} M_{\odot}$. If such an interpretation of the optical - UV emission from $4 \mathrm{C}+01.02$ is correct, the degree of polarization in the optical should be close to zero. This contradicts our SALT spectropolarimetry results which indicate that even in the moderate-activity state in November 2016, the optical polarization is still of the order of $\sim 5-10 \%$. Our combined SED + spectropolarimetry modeling therefore strongly favours a synchrotron-dominated optical emission scenario with the accretion-disk contributing only towards the blue end of the spectrum, requiring a black-hole mass of $M_{\mathrm{BH}} \sim 2 \times 10^{9} M_{\odot}$.

\section{Acknowledgments}

The work of M.B. is supported through the South African Research Chairs Initiative of the South African Department of Science and Technology and National Research Foundation ${ }^{4}$. The authors acknowledge further support by the South African DST through the South African GammaRay Astronomy Programme. The authors are grateful for the support provided by S. Crawford and K. H. Nordsieck with reducing the spectropolarimetry observations. This work makes use of observations from the LCO network. Some of the observations were obtained with the Southern African Large Telescope (SALT).

The Fermi-LAT Collaboration acknowledges support for LAT development, operation and data analysis from NASA and DOE (United States), CEA/Irfu and IN2P3/CNRS (France), ASI and INFN (Italy), MEXT, KEK, and JAXA (Japan), and the K.A. Wallenberg Foundation, the Swedish Research Council and the National Space Board (Sweden). Science analysis support in the operations phase from INAF (Italy) and CNES (France) is also gratefully acknowledged.

\section{References}

[1] Atwood, W. B., et al., ApJ, 2009, 697, 1071.

[2] Atwood, W. B., et al., 2012 Fermi Symposium proceedings, eprint arXiv:1303.3514.

[3] Barres de Almeida, U., et al., MNRAS, 2014, 441, 2885.

[4] Böttcher, M., Ap\&SS, 2007, 309, 95.

[5] Böttcher, M., Reimer, A., Sweeney, K., \& Prakash, A., ApJ, 2013, 768, 54.

[6] Britto, R. J., et al., Proceedings of HEASA2016, Cape Town, South Africa, 2017, PoS(HEASA 2016)021.

[7] Brown, T.M., et al., PASP, 2013, 125, 1031.

[8] Burgh, E. B., et al., Proceedings of the SPIE, 4841 (2003), 1463.

[9] Ghisellini, G., et al., MNRAS, 2011, 411, 901.

[10] Ghisellini, G., et al., MNRAS, 2010, 402, 497.

\footnotetext{
${ }^{4}$ Any opinion, finding and conclusion or recommendation expressed in this material is that of the authors, and the NRF does not accept any liability in this regard.
} 
[11] Marscher, A. P., ApJ, 2014, 780, 87.

[12] Palma, N., et al., ApJ, 2011, 735, 60.

[13] Potter, S. B., et al., Proceedings of SPIE 9908 99082K (August 9, 2016), DOI: 10.1117/12.2232391

[14] Sanchez, D. A. \& Deil, C., In Proceedings of the 33rd ICRC, Rio de Janeiro (Brazil), eprint arXiv:1307.4534.

[15] Rybicki, G. B., \& Lightman, A. P., Radiative Processes in Astrophysics, 1979, John Wiley \& Sons

[16] Shakura, N. I., \& Sunyaev, R. A., 1973, A\&A, 1973, 24, 337.

[17] Smith, P., et al., ApJ, 1986, 305, 484.

[18] Zacharias, N. et al., American Astronomical Society Meeting Abstracts, 2004, 36, 1418

[19] Zhang, H., Chen, X., \& Böttcher, M., ApJ, 2014, 789, 66.

[20] Zhang, H., et al., ApJ, 2015, 804, 58. 